\title{
Vers un modèle du développement vocationnel de l'adulte
}

\author{
DANIELLE RIVERIN-SIMARD, Ph.D.** and JEAN-MARC DION, 1.o.p.***
}

\begin{abstract}
RÉSUMÉ
Cet article présente tout d'abord l'importance et les objectifs de l'étude du développement vocationnel de l'adulte ainsi que son rapport avec les recherches déjà faites et la documentation existante. Les auteurs prétendent que la période adulte (adulthood) a été, à quelques exceptions près, ignorée dans son aspect vocationnel alors que précisément cette connaissance pourrait s'avérer essentielle dans l'élaboration des curricula pour l'éducation permanente. Par la suite, les auteurs présentent le modèle lui-même. Ce modèle postule que le développement vocationnel de l'adulte se poursuit jusqu'à la mort et se réalise par périodes de structuration suivies ou précédées de périodes de transition. Tout en poursuivant son développement vocationnel tout le long de ces périodes, l'adulte vit des périodes de structuration, davantage marquées par une action plus intense, alors que les périodes de transition sont surtout charactéristiques de "questionnement"; ces dernières peuvent conduire à une variété de phénomènes vocationnels incluant, par exemple, des changements radicaux de carrière, des rétrogradations, etc. Chacune des neuf périodes de transition et de structuration ont des contenus bien spécifiques qui les distinquent les unes des autres. Ces diverses périodes (ou stades) sont des passages prévisibles de l'adulte dans sa vie vocationnelle qui donnent un sens, une direction à son évolution ou à son développement vocationnel.
\end{abstract}

\section{ABSTRACT \\ Toward a Model of Adulthood Vocational Development \\ The first part of this article places in its proper context previous research work conducted in vocational development during adult life; this brief overview serves as a yardstick to appraise the lack of importance and general directions given in the literature to this period \\ * La vérification de ce modèle fait présentement l'objet d'une recherche à l'Université} Laval.

** Professeur, Départment de Counseling et d'Orientation, Université Laval

*** Spécialiste de l'animation et de l'encadrement Télé-Université 
of life. The authors argue that continuing education curricula would benefit immensely from greater interest and knowledge derived from the vocational aspects of adults. The second part of this paper presents a model of adulthood vocational development. The model assumes that career development is a life-span process that goes through various structural periods which are either preceded or followed by transition cycles. As vocational development unfolds throughout nine identified stages, adults are usually action-oriented during their structural periods and question-oriented during their transition periods. This constant vocational self-evaluation is the determining force that will give rise to drastic career changes, retrogradation, etc. As each of the nine vocational development periods has distinct characteristics, it becomes possible not only to anticipate them but also to relate them to a particular adult individual's development stage.

\section{Importance et objectifs de l'étude}

Le phénomème de l'éducation permanente, défini comme étant un processus d'éducation qui s'échelonne tout au long de la vie (Hurtubise, 1973; Schwartz, 1973; Houle, 1974) revêt une importance de plus en plus grande dans notre société actuelle (brunner, 1969; Hendrickson, 1960; Linderman, 1961; Kreitlow, 1970; Hurtubise, 1973; Houle, 1975; Nadeau, 1975; Castonguay, 1976). Il s'avère alors essentiel de connaìtre la clientèle de L'éducation permanente que constitue en grande partie les adultes (Desmond, 1956; Vincent, 1961; Birren, 1964a; Birren, 1964b; Vedder, 1765; Bromley, 1966; Time, 1966; Benaim et Allen, 1967; Hahn, 1967; Bischof, 1969; Kroll et autres, 1970). Mais la littérature scientifique concernant l'adulte est très limitée par rapport à la somme de connaissances que l'on possède sur l'enfant et l'adolescent (Schaie, 1973; Clifford et Odin, 1974; Ferland, 1975; Gould, 1975; Nadeau, 1975; Neugarten, 1975; Sheehy, 1977).

Une des approches permettant de procéder à la connaissance de l'adulte qui parait prometteuse et qui n'a pourtant suscité d'intérêt que très récemment est celle de la psychologie développementale (Schaie et Gribbin, 1975; Schaie, 1977). En effet, les données et théoriques de cette approche ont révélé que l'évolution de la personnalité se pursuit au-delà de la cinquième décade et que le processus de formation se pursuit tout au long des étapes da la vie (Super, 1963, 1975; Erikson, 1965; Bunler, 1968; Crites, 1969; Havighurst, 1969; Huberman, 1974; Gould, 1975; Levinson et autres, 1976; Lerner, 1976; Sheehy, 1977). Cette approche s'est révélée d'une grande importance en ce qui concerne l'enfant et l'adolescent car les données qu'elle a fournies ont le mérite d'avoir éclairé la nature de leur développement; cette connaissance a permis que l'éducation de ces enfants et de ces adolescents puisse être davantage conçue et vécue comme un processus continu tenant compte de la totalité de l'être (Tryon et Lilienthal, 1950; Ginzberg, 1956; Erikson, 1968; Havinghurst, 1972; Prince, 1973; Super, 1975).

Comme le souligne Parker (1974), après avoir fait un relevé des écrits pertinents, la psychologie développementale, parce qu'elle se préoccupe de la direction, du niveau et du contenu du changement, offre de grandes possibilités pour indiquer le sens à donner aux processus d'éducation. En effet, en spécifiant les comportements particuliers qui sont caractéristiques d'un stade donné chez la personne, on est capable d'élaborer des stratégies d'intervention ou un curriculum en éducation facilitant le passage de ce stade et ainsi 
activer le développement ou l'évolution de cette même personne. Et c'est précisément ce qui apparait comme nécessaire dans l'élaboration des curricula pour l'éducation des adultes (Becker, 1964; Neugarten, 1965; Brim et Wheeler, 1966; Kreitlow, 1970).

Il nous a dès lors semblé à la fois très pertinent et urgent de s'intéresser à une avenue plus spécifique de la psychologie développementale, à savoir l'étude du développement vocationnel de l'adulte.

On définit généralement le développement vocationnel comme un processus qui se compose de differents événements séquentiels tout le long d'une carriére et durant la retraite. Le déroulement de ce processus est généralement ordonné et prévisible; il est également dynamique en ce sens qu'il résulte de l'interaction entre les aspirations de l'individu et les demands de la culture; au cours de ce processus, l'individu doit s'acquitter d'un certain nombre de tâches dites "vocationnelles". Dans cette prespective, l'avenue spécifique de l'étude du développement vocationnel de l'adulte apparait d'autant plus prometteuse que la formation et le perfectionnement professionnels sont encore à l'heure actuelle une des raisons majeures qui poussent les adultes à fréquenter les institutions scolaires et s'avèrent également l'essentiel même du contenu des curricula (Charters, 1970; Schwartz, 1973; Castonguay, 1976). Il faut donc croire que les phénomènes de l'éducation permanente et du développement vocationnel de l'adulte sont en liaison très étroite, le premier étant un des moyens privilégiés de concrétiser et d'activer la réalisation du second.

De plus, il n'y a que quatre(4) auteurs à date qui se sont intéressés au développement vocationnel chez les adultes (Super, Miller et Form, Havinghurst, Tiedeman et O'Hara). Parmi ces auteurs, seul Super peut être considéré comme ayant traversé la phase embryonnaire des travaux de recherche. Mais, tant dans l'explication des stades et sous-stades, Super, qui avait formulé sa théorie dans les années 1950 , ne traite à peu près pas de phénomènes vocationnels aujourd'hui plus courants ou plus répandus ou s'il en traite, il les explique en termes de phénomènes malsains et instables. Ces phénomènes plus courants aujourd'hui que vers les années 1955 sont les suivants: la retraite prématurée, les changements radicauz de carrière, la rétrogradation volontaire des divers cadres, les périodes moratoires de plus en plus exigées par les conventions collectives (années sabbatiques à divers paliers des travailleurs spécialisés), l'abandon de la permanence d'emploi chez plusieurs spécialistes qui préfèrent louer leurs services pour de courtes périodes, une conception du travail comme un simple moyen de pouvoir se permettre certaines activités de loisirs ou certaines activités civiques, etc. (Toffler, 1970).

Ainsi, étant donn-e d'une part l'importance de l'éducation permanente et l'importance de la connaissance du développement personnel et vocationnel de l'adulte et, étant donné d'autre part l'état de la littérature pertinente, il s'avère d'autant plus nécessaire de procéder à l'étude du développement vocationnel de l'adulte d'aujourd'hui.

\section{Rapport avec les recherches déjà faites et avec la documentation existante}

La conception fondamentale de l'éducation permanente sur laquelle la présente étude s'appuie correspond à un des trois courants théoriques identifiés par Houle $(1969,1970)$. Selon ce courant, l'éducation est un processus continu qui varie selon les divers stades de la vie. Cette conception rejoint de très près celle de la psychologie développementale qui veut que l'individu soit un être éducable défini comme une personne qui possède des 
possibilités de développement et d'auto-réalisation (Erikson, 1965; Chickering, 1969; Havighurst, 1972; Super, 1975; Lerner, 1976).

Même si théoriquement, les deux conceptions fondamentales sur lesquelles se base cette étude se rejoignent de très près, il est alors surprenant de constater que, parmi les études relatives à l'éducation des adultes, aucune n'est basée sur les schémas théoriques de la psychologie développementale pour étudier le phénomène de l'éducation permanente. En effet, les différents relevés de littérature ne font aucune mention d'études de ce genre. Du côté de la psychologie développementale certains auteurs dont Super $(1957,1975)$, O'Hara et Tiedeman (1959), Bühler (1968), Pikunas (1969), Havighurst (1972), Houle (1974), Gould (1975), Hurlock (1975), Kaluger (1976), Levinson (1976), Rappoport (1976), Vaillant (1977) se sont intéressés à l'adulte mais ces recherches sont encore à l'état embryonnaire (Clifford et Odin, 1974; Gould, 1975; Levinson, 1976; Sheehy, 1977).

Ainsi d'une part, si théoriquement l'éducation permanente est un processus continu qui varie selon les divers stades de la vie et que, d'autre part, empiriquement, on ne connaît à peu près pas les stades de la période adulte, il devient donc nécessaire de procéder à l'identification des stades de développement personnel et vocationnel de l'adulte afin de mieux concevoir la dynamique du processus de l'éducation permanente et de planifier des activités qui se veulent non seulement respectueuses de ce développement, mais surtout susceptibles de l'activer.

Devant la rareté de la littérature théorique (Goulet et Baltes, 1970; Wohlwill, 1970; Schaie, 1973; Schaie et Gribbin, 1975), il est apparu nécessaire de construire en une première étape, un modèle théorique du développement vocationnel de l'adulte. Ce modèle, qui est strictement un modèle organique de développement selon la nomenclature de Overton et Reese (1973), est la conception des auteurs du présent article. Ce modèle a été conçu à partir de la littérature existante sur le développement personnel de l'adulte et à partir des expériences de pratique de la psychologie et de l'orientation scolaire et professionnelle du premier auteur cité (pratique professionnelle de 9 ans auprès des étudiants de collège et des adultes au Collège SteFoy, P.Q.). Et, parallèlement à Super qui s'est surtout basé sur la théorie de Bühler pour concevoir sa théorie du développement vocationnel, les auteurs du modèle se sont surtout basés sur deux modèles récents du développement personnel (sens général du terme, en touchant à peu près pas au développement vocationnel) de l'adulte. Ces modèles sont ceux de Gould (1975) et de Levinson (1976). Ces deux modèles comportaient une perspective tenant nettement plus compte des nombreuses possibilités d'évolution et de développement de l'adulte aux diverses périodes de la vie, tandis que le modèle de Bühler, basé surtout sur l'évolution biologique de l'individu, avait inspiré à Super une conception du développement vocationnel par des stades d'établissement, de maintenance et de déclin, perspective davantage déterministe qui laissait moins de place à la possibilité d'un développement vocationnel différent mais constant de l'individu aux diverses périodes de la vie.

\section{Les grandes lignes du modèle}

Le développement vocationnel de l'adulte se poursuit jusqu'à la mort et se réalise par périodes de structuration suivies ou précédées de périodes de transition. Tout en poursuivant son développement vocationnel tout le long de ces périodes, l'adulte vit des périodes 
de structuration, davantage marquées par une action plus intense, alors que les périodes de transition sont surtout caractéristiques de "questionnement"; ces dernières peuvent conduire à une variété de phénomènes vocationnels incluant, par exemple, des changements radicaux de carrière, des rétrogradations, etc. Chacune des neuf périodes de transition et de structuration ont des contenus bien spécifiques qui les distinguent les unes des autres. Ces diverses périodes (ou stades) sont des passages prévisibles de l'adulte dans sa vie vocationnelle qui donnent un sens, une direction à son évolution ou à son développement vocaitonnel.

Les stades de transition permettent de préparer un changement, de plus ou moins grande importance, de modalité de vie vocationnelle, i.e. des configurations particulières "personnalisées" d'actualisation de divers rôles vocationnels. Les stades de structuration se présentent plutôt comme le moment de concrétiser ce changement.

Les postulats généraux transcendant notre modele sont basés sur une conception organique de l'évolution de l'adulte. Un premier postulat veut que la personne a des poussées intrinsèques de développement, d'évolution constante et ce, jusqu'à la mort, différemment de la courbe biologique. Et ce développement vocationnel et l'adulte, même si moins "apparent" que durant l'enfance et l'adolescence est tout aussi intense et ramifié et peut même se spécifier par tranche approximative de cinq (5) ans. Le temps, où se situent les événements intérieurs et extérieurs que vit l'individu, provoque des dissonances qui amènent l'individu à vivre des périodes de questionnement (transition) pour mieux se stabiliser temporairement, pour mieux se resituer, se restructurer (structuration). Et ainsi de suite, durant les périodes de structuration, le temps provoque, de par les événements qui l'accompagnent, d'autres périodes de questionnement.

Les événements extérieurs "amenés" par le temps seraient des phénomènes courants typiques à un âge donné. Par exemple, la vie commune, le départ des enfants du foyer, l'âge social de la "retraite" sont des phénomènes plus ou moins vécus universellement par les différentes cultures. Ainsi les événements "extérieurs" dont on fait mention dans la présentation du modele ne contiennent pas des événements "extra-ordinaires" comme les guerres, les cataclysmes ou autres événements majeurs du genre. Car à ce moment, la variable "génération" viendrait interférer l'aspect universel du modele.

Le modèle utilise l'âge comme étant intrinsèque au développement. En ce sens que ce n'est pas l'âge qui provoque une transition ou une restructuration (perspective où l'on utiliserait l'âge comme étant intrinsèque au développement, Lofft (1973). Ce sont les événements vocationnels (tant de son monde intérieur qu'extérieur) véhiculés dans le temps qui provoquent la poussée constante de l'évolution ou du développement vocationnel. Dans le présent modèle, l'âge ne s'avère donc qu'un index en ce sens qu'il n'est pas nécessairement inhérent à tous les comportements observés.

Le modèle n'inclut pas la notion de régression comme tel dans le développement vocationnel, mais plutôt des périodes de remises en question, des moments d'arrêt (transition) pour permettre une évolution plus intense, plus rapide dans la période subséquente (structuration). En ce sens le modèle présenté ici rejoint les autres théories organismiques qui ne parlent pas de "réelles" périodes de régression mais plutôt des périodes d'instabilité précédées ou suivies d'un temps d'équilibre (Looft, 1973).

De plus, le modele présenté conçoit le développement vocationnel comme étant simultanément unidirectionnel et multidirectionnel (nomenclature de Looft, 1973). Le 
développement est unidirectionnel car l'individu continue de progresser, ne repart jamais à zéro malgré d'apparentes régressions. L'individu traversera le stade subséquent indépendamment de sa réussite ou non avec la négociation des tâches du stade précédent. Et ce, parce que ce sont les événements véhiculés par le temps, événements de son monde intérieur ou extérieur, qui constituent la poussée interne de développement et qui provoquent le cheminement caractérisé par une évolution constante.

Le développement vocationnel est également conçu dans le modèle présenté ici, comme étant multidirectionnel. En effet, un individu peut poursuivre, pendant un ou plusieurs stades, son évolution dans un contexte vocationnel et selon une modalité de vie vocationnelle " $x$ " et, après un stade de transition, poursuivre son développement dans un sens complètement différent et "apparemment" imprévisible ou même contradictoire d'avec le stade précédent. Cet aspect du développement vocationnel multidirectionnel est à l'opposé de la notion de Super (1963), qui voit le développement vocationnel de l'adulte comme étant une suite "logique" "séquentielle" d'actes professionnels et de vie vocationnelle à l'intérieur (ou à peu près) d'un même champ professionnel ou d'un même domaine vocationnel.

Le modèle postule que les stades de transition et de structuration sont des aspects universels du développement vocationnel de l'adulte. Chaque individu évolue selon une séquence invariante de stades développementaux.

Enfin, dans les grandes lignes du modèle que les présents auteurs proposent, il y a toutes les notions reliées de "modalité de vie vocationnelle", de "changement de modalité de vie vocationnelle", de "degrés de changement de vie vocationnelle", de "types de développement vocationnel".

Etant donné que le modèle présenté inscrit essentiellement des périodes successives de transitions et de structuration, il postule implicitement que le développement vocationnel qui se poursuit tout au long de la passation des stades est caractérisé par des changements de modalité de vie vocationnelle. Ces changements qui peuvent être de différents degrés, sont des modifications de modalité de vie vocationnelle. Ces modifications sont préparées durant les stades de transition et sont vécues ou concrétisées durant les stades de structuration.

Une modalité de vie vocationnelle est une forme particulière d'actualisation des divers rôles vocationnels tenant compte de leurs différents facteurs. Ces facteurs peuvent être d'ordre extrinsèque comme le champ professionnel, le niveau hiérarchique dans les champs professionnels, la nature de l'occupation, le niveau de spécialité requis pour la tâche, l'employeur, la nature de la différence des tâches à l'intérieur d'un même champ professionnel, la nature de la similarité des tâches dans des champs professionnels différents, etc. Ces facteurs peuvent être d'ordre intrinsèque comme la façon personnelle d'aborder les tâches, la perception de l'utilité de ces tâches, la réorganisation hiérarchique de l'importance des tâches à accomplir, etc.

Pour mieux saisir ce que le modèle entend par changement de vie vocationnelle, on peut donner un exemple d'une modification selon un degré élevé. Une personne qui pratique à titre de conseiller d'orientation dans une école et qui, après une période de questionnement ou après avoir traversé un stade de transition décide d'organiser sa propre entreprise florale. Une personne qui a un doctorat ès lettres et qui est affectée à des tâches de recherche à un niveau universitaire et qui, après une période de questionnement, décide de devenir fermier. 
7 Vers un modèle du développement vocationnel de l'adulte

Les deux (2) cas précités sont des exemples de changements de modalités de vie vocationnelle à un degré élevé. En effet, dans les deux (2) cas de nombreux facteurs ont été touchés: les employeurs ne sont plus les mêmes, ni la nature des tâches, ni le niveau ou la nature de spécialité requis; il n'y a à peu près pas de similarités entre les tâches précédentes et les tâches actuelles; la réorganisation hiérarchique de l'importance des tâches à accomplir a été plutôt radicale.

Quant aux degrés de changement de modalités de vie vocationnelle, on pourrait arbitrairement et temporairement en identifier une dizaine qui irait du degré de changement le moins élevé au degré de plus élevé. Par exemple, un changement au premier degré est un changement qui implique des modifications dans l'un des facteurs de modalités de vie vocationnelle énumérés dans les paragraphes précédents. Par exemple, un changement au dixième degré impliquerait des modifications dans l'ensemble des facteurs de vie vocationnelle.

Dans le modèle de développement, l'évolution permanente étant un postulat, il y a toujours entre deux (2) stades de structuration, au moins un changement de premier degré de vie vocationnelle. Et, étant donné que le développement vocationnel de l'adulte s'inscrit à la suite du développement vocationnel de "l'adolescence", le modèle postule qu'il y a eu, juste précédant le stade de structuration I, un stade de transition. Ainsi, le modèle postule que l'entrée au stade de structuration I comprend, au moins, un changement au premier degré de modalité de vie vocationnelle en comparant cette modalité à celle existant lors du stade précédant la période dite "adulte".

Le modèle indique également des "types de développement vocationnel" fondés sur l'ampleur et la continuité des modifications de modalités de vie vocationnelle. Ces types de développement sont autant de modes de développement "sain", "évolutif", qui ne sont pas, aux extrêmes, considérés comme soit des types de développement instables ou "maladifs". Il y a quatre (4) types de développement vocationnel: il y a le type "silencieux", le type "accidentel", le type "zigzaguant" et le type "spectaculaire".

Le type "silencieux" de développement vocationnel durant la période adulte serait celui d'un individu qui vit des changements minima de modalité de vie vocationnelle (changements au premier ou deuxième degré).

Le type "accidentel" de développement vocationnel durant la période adulte serait celui d'un individu qui, dans l'ensemble vit des changements de modalité de vie vocation. nelle surtout minima mais qui, à la suite d'un ou de deux changements allant du septième au dixième degré.

Le type "zigzaguant" de développement vocationnel durant la période adulté serait celui d'un individu qui vit des changements de modalité de vie vocationnelle tantôt dans les premiers degrés, tantôt dans les derniers degrés ou tantôt dans les degrés intermédiaires.

Le type "spectaculaire" de développement vocationnel durant la période adulte serait celui d'un individu qui vit des changements de modalité de vie vocationnelle surtout maxima, i.e. se situant presque toujours ou toujours dans les changements de degrés élevés.

\section{Le Modele}

\section{- Premier Cycle (23-42 ans)}

S.I. - Stade de structuration initiale de l'identité vocationnelle de l'identité vocationnelle de l'adulte (23-27 ans) 
Ce premier stade, dit de structuration, est celui du choix d'un employeur et marque le début d'un engagement dans une identité vocationnelle. L'adulte de la vingtaine étant devenu potentiellement productif, au sens où la société le conçoit, s'engage à essayer de répondre à cette attente de la société.

Le jeune adulte cherche avant tout à se choisir une occupation mais aussi à s'établir dans une direction vocationnelle consistante avec ses intérêts, ses valeurs et son "image de soi". Il emploie donc la majorité de son énergie à essayer de réaliser ce moi défini au cours des années antérieures; autrement dit, il èssaie de concrétiser son choix vocationnel.

A son point de vue, l'identité qu'il s'était défini, et qu'il essaie de concrétiser pour la première fois, est bien établie, même si elle ne le satisfait pas pleinement. Aussi, il ne dépensera que peu d'énergie à considérer le bien-fondé de son engagement et à s'interroger sur le sens de ses actions professionnelles car, pour lui, ses actions sont non seulement justifiées, mais elles sont à ce moment les seules qu'il juge adéquates.

La tâche développementale vocationnelle principale de cette période rëside donc dans l'exploration des possibilités du monde adulte afin d'en arriver à:

1. se confronter pour la première fois à une définition de soi en tant qu'adulte travailleur; 2. se former une structure initiale de vie vocationnelle qui lui permettra de se reconnaitre et de s'estimer en tant qu'adulte.

A cet égard, le rôle des parents est encore important, ne serait-ce que pour permettre à l'adulte de cet âge de prouver à ceux-ci sa compétence comme adulte travailleur et ainsi de se définir comme adulte capable de se faire une place sur le marché du travail, de subvenir à ses besoins et de se réaliser sur le plan occupationnel dans le sens de l'identité choisie.

Le rôle des collègues de travail est aussi important; ils ont toutefois un impact relatif sur l'évaluation ou la remise en question de ses manières de réaliser les tâches professionnelles, cette évaluation n'étant pas prioritaire à cette période-ci.

Un concept-clé dans l'analyse de ce stade est celui de rêve en ce sens que même sa perception du présent se fait en regard du futur à construire. La plupart des gens, sinon tous, entrent sur le marché du travail avec, en tête, un rêve ou une vision de leur propre futur vocationnel; ce peut-être: gagner un prix Nobel, contribuer de quelque façon au bien-être humain, etc.

L'individu explore la nature et les déroulements de ce rêve tout le long de sa vie. La majorité des changements dans la direction vocationnelle, à des âges subséquents, sont occasionnés pour une réactivation dans le sens d'une redécouverte de ce rêve ou de la réalisation d'un compromis de ce rêve.

Les crises des années 30,40 , etc., se traduisent souvent par un phénomène majeur dont les deux pôles sont:

1. la réactivation d'un rêve-guide, découlant de la période de l'adolescence ou du début de la vingtaine;

et

2. la crainte de ne pouvoir réaliser ce rêve.

Enfin, ce stade, comme tout autre stade qui consiste à s'introduire dans un nouveau cycle de vie (tels la période "jeune adulte", l'âge mûr, la retraite), est un temps de premier choix et d'essai. Somme toute, ce stade est un premier essai de concrétisation d'une identité vocationnelle adulte et entretien d'un rêve vocationnel pour les années ultérieures. 
9 Vers un modèle du développement vocationnel de l'adulte

\section{T.I - Stade de transition: Questionnement sur d'autres modalités de réalisation de l'identité vocationnelle (28-32 ans)}

L'adulte de ce stade dit de transition vit une expérience totalement différente du monde du travail. Alors que celui du stade précédent se remettait peu en question et se donnait comme tâche essentielle l'actualisation de son identité, l'adulte de 28-32 ans est porté à se questionner: il remet en question le mode d'actualisation de ce moi vocationnel en se demandant si c'est là l'unique façon de se réaliser. Tout en continuant malgré tout cette actualisation, l'adulte ne cherche qu'à être ce qu'il est sur le plan occupationnel, et non plus être ou tenter d'actualiser ce qu'il croyait être. Il s'aperçoit en effet que ce qu'il croyait être n'était en effet qu'une des modalités possibles d'actualiser son identité.

Ce stade prend forme au moment où certains besoins ou intérêts font leur apparition dans un plan de carrière pourtant bien établi. L'adulte de ce stade ne se satisfait plus de se consacrer uniquement à la tâche d'actualiser un choix vocationnel effectué au cours des années antérieures. Et, d'une façon particulière, il y a une "conscientisation" naissante du fait que sa volonté de réaliser certains aspects (plutôt sociaux) du moi vocationnel ne l'a pas complètement satisfait; des forces internes le poussent à vouloir reproduire d'autres aspects du moi qui ne sont pas encore actualisés et même s'il ne le voulait pas nécessairement, il ne pourrait désormais ni les ignorer, ni les rejeter.

Ce stade de transition en est donc un à la fois de "questionnement" et de découverte: "questionnement" sur des modalités de se réaliser sur le plan occupationnel et de découverte de besoins internes à actualiser.

Par rapport à ses parents, l'adulte de 28-32 ans se sent plus libre de reconnaître et d'accepter des similitudes entre son cheminement vocationnel et ces derniers. Il ne voit plus la nécessité de prouver sa compétence ou de mettre en évidence ses différences ou son originalité, comme c'était le cas auparavant.

L'illusion de toute-puissance, telle qu'elle était vécue au stade précédent, s'amenuise graduellement. Elle fait place à l'émergence d'une identité vocationnelle transormée dont l'installation définitive se fera au stade suivant.

Le stade de 28-32 ans peut donc occasionner de considérables agitations et remises en question, tant en regard du milieu que de soi. Il peut également se vivre par une réévaluation plus calme et une intensification de l'effort d'actualisation vocationnelle mais il est marqué par des changements importants dans la structuration initiale de l'identité vocationnelle.

Enfin, ce stade, comme tout stade de transition, permet de considérer d'autres avenues possibles de développement vocationnel et de s'engager dans celle que l'individu juge la plus adéquate.

\section{S.II - Stade de structuration: engagement vocationnel profond (33-37 ans)}

$\mathrm{Au}$ cours de ce stade, l'adulte, qui vient de passer une étape de transition, de "questionnement", s'engage beaucoup plus profondément et de façon plus personnelle qu'au stade de structuration précédent. Au cours de cette seconde structuration, il se trace des plans et des buts à plus long terme. Il s'investit davantage dans son travail, celui-ci étant souvent vu comme un espoir de compensation face à la fuite du temps, face aussi à un besoin de prouver sa réussite. 
L'adulte voit que, sur le plan occupationnel, il aurait peut-être une dernière chance de faire sa marque et de s'établir une structure occupationnelle ultime et durable. Et on remarque qu'à partir du milieu de ce stade, soit vers 35 ans, l'adulte cherche désespérement à s'affirmer dans les rôles les plus valorisants pour lui: il tente de vivre des événements-clés dans sa carrière, dans l'optique évidente de laisser le message ultime d'un être de valeur.

Une promotion ou un changement de travail revêtira une importance majeure au cours de ce stade.

L'adulte de ce stade doit composer avec deux aspects antithétiques de son développement vocationnel: d'une part, un désir d'ordre, de sécurité, de stabilité et de contrôle; de l'autre, un goût de réussite et d'ascension occupationnelle. En effet, s'établissant dans la société et poursuivant ses intérêts d'après un "pattern" bien défini, il doit du même élan planifier ses actes professionnels en vue d'élaborer un calendrier d'opérations pour mener à terme la réalisation de ses plans occupationnels.

Alors que de 23 à 27 ans, la perception du temps se faisait à l'égard du futur à construire, cette même perception est maintenant marquée par le présent à occuper pleinement. Ce début de "conscientisation" du temps comme limite amène comme conséquence l'obligation de se restreindre dans les choix ou possibilités de travail, de songer aux ressources pécuniaires et au bien-être personnel. 11 y a aussi une prise de conscience de certaines limites physiques et celle d'un potentiel psychologique illimité et insoupçonné.

La création de ce stade de structuration, qui se veut intégrée, ne va donc pas sans heurts, car l'individu ne peut utiliser que des parties de son moi vocationnel au détriment d'autres. Mais, en définitive, cette période se vit sous le signe de l'épanouissement vocationnel.

$\mathrm{Au}$ cours de ce stade, le rôle du mentor s'avère prépondérant; car l'engagement profond vécu à ce stade est souvent le résultat des conseils et encouragements prodigués par le mentor au jeune adulte.

La présence du mentor est toutefois une composante importante dès le premier stade de structuration de l'identité vocationnelle adulte; elle n'est donc pas exclusive au stade 33-37 ans.

Ce mentor a habituel lement 8 à 15 ans de plus que le "mentee". C'est soit un professeur, un patron, un éditeur ou un collègue de travail expérimenté. Il accélère son développement vocationnel en le guidant dans le monde professionnel et occupationnel; en retour, ce dernier lui apporte gratitude, admiration et respect.

Cette relation prend souvent fin avec le stade suivant i.e., vers 37-38 ans. Après ce stade, l'adulte n'est plus “mentee".

\section{T.II - Stade de transition: rétrospective - prospective ou période moratoire (3842 ans)}

Vers 37 ans, l'adulte pensait que s'il obtenait ce qu'il voulait, tout serait structuré de façon définitive. Mais à mesure qu'il approche de la quarantaine, il se rend compte que tout ne se vit pas comme prévu. Une des raisons pour lesquelles le stade précédent doit changer, c'est que ce stade est jusqu'à un certain point basé sur des illusions, illusions sur l'importance et le sens suivant lequel réaliser ses objectifs vocationnels et entretenir des relations avec des relations avec des personnes significatives sur le plan occupationnel pour poursuivre ces objectifs.

Un processus de désillusionnement (par lequel on doit signifier une diminution ou un réaménagement des illusions par opposition à une désillusion cynique) devient un aspect 
11 Vers un modèle du développement vocationnel de l'adulte

important du développement vocationnel de l'adulte de ce stade. Il s'interroge alors sur son moi, ses valeurs et sur sa vie vocationnelle en général.

Mais, contrairement au stade de transition précédent, les questions ont davantage une tendance ou une connotation de "désespoir tranquille" et une conscience accrue de la fuite du temps. Ces questions touchent de très près son cheminement vocationnel. Ainsi, au lieu de se dire: "Je veux être comme je suis", dans un sens d'infinité du temps, l'adulte se demande: "Ai-je vraiment fait le bon choix? et "Est-il encore temps de changer?"

Ce stade de transition peut-être plus ou moins difficile à traverser; mais il survient, peu importe que l'individu réussisse ou échoue dans sa recherche d'affirmation de son identité. La réussite ou l'échec ne sont pas ici considérés par rapport au nombre de récompenses (ou gratifications) reçues mais plutôt face à l'adéquation entre sa structuration vocationnelle et l'identité vocationnelle; car un adulte peut très bien réussir dans l'atteinte de ses objectifs vocationnels et, malgré tout, vivre ces succès de façon passablement décevante. Il est donc confronté à la nécessité de traverser ce stade de transition, peu importe que cette affirmation d'une identité vocationnelle ait été réalisée ou pas.

Les questions suivantes se posent, questions qui supposent un mouvement vers l'intériorité: "Qu'ai-je atteint à ce moment de ma carrière?" et “Qu'est-ce que je veux réellement réaliser sur le plan occupationnel?". C'est donc un moment central pour faire le point sur le plan occupationnel et vocationnel, car ces questions, qui accentuent la limite du temps, comportent un regard simultané vers le passé, le présent et l'avenir.

Comme tout stade de transition, celui-ci place l'adulte dans une situation de choix possibles de structuration. Ainsi, entre 40 et 42 ans, se pose un choix à faire entre le bien-être matériel et le bien-être personnel.

Par exemple, l'adulte de cet âge peut avoir à résoudre le compromis suivant: demeurer dans un emploi suffisamment rémunérateur et relativement satisfaisant, ou songer à changer d'emploi ou même d'orientation, changement qui lui permettrait de réaliser le nouveau moi vocationnel, même si cela se produit dans un climat momentané d'inconfort, d'instabilité et d'incertitude.

\section{Deuxieme Cycle (43-62 ans)}

\section{S.III - Stade de structuration: mouvement vers l'intériorité (43-47 ans)}

Suite aux questions du stade précédent, l'adulte prend davantage conscience, et avec plus de force que jamais, de ses intérêts vocationnels négligés tout au long de sa carrière ainsi que de certains besoins vocationnels insatisfaits et qui reviennent implacablement à la surface. On eput qualifier ce stade de développement vocationnel comme un effort de vivre cette identité d'une manière plus personnelle en tenant davantage compte de ses impératifs internes que des exigences externes.

Un nouveau stade de structuration prend donc forme, structuration axée sur un mouvement vers l'intériorité; et cette structuration se veut une période d'exploration du deuxième cycle, tout comme le stade de 23 à 27 ans en regard du premier cycle.

L'adulte de cet âge vit maintenant avec les résultats anticipés antérieurement. Sur le plan occupationnel, ce stade fait prendre durement conscience que, le temps s'écoulant rapidement, il devient difficile de songer à des modifications majeures ou à des exploits occupationnels. 
De plus, la place faite à des aspects négligés du moi vocationnel l'est souvent au détriment d'une réalisation de carrière éclatante, carrière que l'adulte commence à trouver moins importante parce que temporaire.

L'adulte de ce stade ressent que sa personnalité est bien établie et que, pour employer les termes anglais "The die is cast" (le moule est coulé). Le fait de vivre avec ce qu'il est peutêtre une pilule amère à avaler mais, du même coup, c'est un soulagement face aux dépassements qu'il se sentait obligé de réaliser.

Face aux collègues de travail, surtout à l'égard des plus jeunes, il aura une tournure d'esprit plutôt négative et compétitive. La raison principale est qu'il se sent poussé par ces jeunes adultes désireux de réussir et de laisser leur marque. Ce qui précède est marqué par la prise de conscience du vieillissement, forçant l'adulte à se définir dans la catégorie des vieux plutôt que dans celle des jeunes. D'ailleurs, les archétypes de puérilité et de sénélité jouent d'une façon importante dans le processus exploratoire de ce mouvement vers l'intériorité. Somme toute, l'adulte de cet âge essaie de vivre son identité vocationnelle en tenant compte des aspects autrefois négligés de son moi.

\section{T.III - Stade de transition: Questionnement sur une nouvelle modalité de vie vocationnelle faite de compromis (48-52 ans)}

Le déclin physique et l'implacable éventualité de la mort sont deux thèmes qui forçent l'adulte de ce stade à se redéfinir. L'adulte en est donc à un stade de "questionnement" sur une façon de réaliser le compromis suivant: d'une part, continuer à vivre sa vie vocationnelle d'une façon surtout individualiste, en se préoccupant essentiellement de ses valeurs au détriment des valeurs occupationnelles du monde extérieur; d'autre part, devant une découverte plus intense, caractéristique de ce stade, des détresses d'autrui, vivre un cheminement vocationnel axé sur l'humanisation de ses actes occupationnels et la compassion envers ses collègues de travail.

Les réponses à ce "questionnement" sur les actualisations possibles du moi vocationnel se feront, au stade suivant, en regard de cette redéfinition existentielle faite surtout en fonction d'autrui. Ce stade apporte donc des choix nouveaux à vivre, et ces choix ne se situent pas tant au niveau du contenu occupationnel de la carrière mais à celui de relations de travail, bien que les deux interagissent inévitablement. Somme toute, l'adulte de cet âge vit une période de "questionnement" sur la possibilité de vivre une identité vocationnelle d'une façon simultanément plus individualiste et plus humanisante envers son milieu de travail.

\section{S.IV - Stade structuration: engagement vocationnel serein (53-57 ans)}

L'adulte de ce stade, sentant moins la nécessité de se confronter aux nouvelles connaissances techniques relatives à son travail et par là, moins le besoin de se battre pour se maintenir à jour, vit une période moins agressive, plus positive et chaleureuse. La compétition parfois difficile des jeunes années est tempérée par une meilleure connaissance de soi et, son individualité étant habituellement moins menacée, il a des relations plus détendues avec ses collègues de travail.

Ce stade, aussi important que les précédents sur le plan du développement vocationnel, fournit l'occasion d'un engagement personnel très intense dans le domaine occupationnel, bien que cet investissement soit empreint de détachement et de calme. C'est le moment 
13 Vers un modèle du développement vocationnel de l'adulte

où fort de son expérience, l'adulte peut contribuer de façon consultative à l'avancement et au progrès. On écoute facilement ses conseils sur le plan travail, d'autant plus qu'il ne les donne pas pour faire réagir les gens ou obtenir leur approbation, devenant moins sensible à une telle réaction d'autrui. Bref, ce stade en est un d'influence majeure.

Cette sérénité externe va de pair avec une préoccupation accrue de la vie intérieure. S'il ne tient pas nécessairement à relever d'autres défis, bien qu'il en soit capable, l'adulte de ce stade trouve un grand plaisir dans son travail, en privilégiant ce qui l'intéresse.

\section{T.IV - Stade de transition: bilan de la carrière "active" et questionnement sur des modalités de vie vocationnelle de "retraités" (58-62 ans)}

L'adulte de ce stade dresse un bilan, une rétrospective de ses contributions au monde du travail. Cette rétrospective se fait surtout à partir des années 50 , i.e., au début du stade d'engagement vocationnel et social plus altruiste. Par ailleurs, certaines inquiétudes se font jour: le temps disponible semble toujours se retrécir et la présence de la mort devient imminente.

Sur le plan occupationnel, l'adulte se demande si, malgré le système socio-économique qui le pousse à se retirer, à devenir moins efficace et moins productif, il ne pourrait quand même pas planifier une modalité différente d'apport au monde du travail.

Cette interrogation fait suite à la découverte d'une philosophie de la productivité qui veut que soient dépassées les vieilles notions suivant lesquelles l'adulte doit à cet âge ralentir ou abandonner.

L'adulte aimerait se planifier de nouveaux "horizons" vocationnels. Les questions suivantes se posent: "Puis-je encore me réaliser dans des activités occupationnelles? Puis-je encore me développer dans un travail, et ce, jusqu'à la mort?"

L'adulte du début de la soixantaine cherche surtout à découvrir les modalités d'activités qui lui permettraient de poursuivre son évolution vocationnelle et personnelle: "Que vais-je faire durant ma retraite?"

\section{- Troisieme cycle (63 - ...)}

\section{S.V - Stade de structuration: essai de sa nouvelle modalité de vie vocationnelle en tant que "retraité" (63-67 ans)}

Fonction des réponses apportées aux questions du stade précédent, l'adulte poursuivra son développement vocationnel selon une modalité adaptée à son statut de retraité. Se trouvant libéré et "désengagé" des préoccupations occupationnelles (travail à temps plein) qui conviennent mieux à des gens plus jeunes, il lui devient possible de s'engager dans des activités plus personnalisées.

Etant libéré des contraintes occupationnelles passées, il peut, s'il le désire; s'investir davantage dans une "production" correspondant à ses intérêts et ses possibilités actuelles. Cette production, dans laquelle l'adulte de ce stade s'investit, peut prendre diverses formes, allant du travail rémunéré à tous les substituts de l'activité-travail.

L'important pour l'individu, au cours de ce stade, est d'explorer cette nouvelle modalité de vie vocationnelle. Comme pour tout autre stade d'essai, cette nouvelle modalité n'a pas un caractère définitif. 
14 Danielle Riverin-Simard and Jean-Marc Dion

\section{T.V, S.VI, T.VI . . - - Stades allant vers l'infini}

Sans donner de précision sur le contenu de ces stades ultérieurs, le modèle postule une possibilité infini de cette rotation de stades de transition et de structuration se terminant avec la vie biologique de l'individu.

\section{Conclusion}

Etant donné l'importance de l'étude du développement vocationnel, les auteurs du présent modèle croient que le modèle présenté devrait faire l'objet de vérification auprès de la population adulte. Cette vérification ou cette recherche serait d'autant plus importante si l'on songe aux implications théoriques et pratiques qui en découleraient.

Sur un plan théorique, cette recherche permettra d'élucider les conceptions de l'éducation permanente au moyen de celle de la psychologie développementale. De plus, elle offrira un modèle théorique pouvant être à la base de projets de recherche ultérieurs, modele nécessaire étant donné l'état actuel de la littérature dans le domaine du développement de l'adulte (Schaie, 1973, 1977), (Wohlwill, 1970), (Goulet et Baltes, 1970), (Schaie et Gribbin, 1975).

La portée pratique du présent projet consistera dans les points suivants: 1 . connaissance de la clientèle, actuelle et éventuelle, de l'éducation des adultes; 2 . données pouvant permettre l'élaboration d'une meilleure politique d'expansion des services d'éducation permanente et par là une planification toujours plus rationnelle et systématique de leurs diverses activités; 3 . données permettant aux praticiens de l'enseignement aux adultes et au personnel de support (conseillers pédagogiques, conseillers d'orientation, travailleurs sociaux, etc.) de planifier une intervention plus adéquate auprès des adultes.

Enfin, cette recherche permettrait à l'éducation des adultes à mieux s'insérer dans l'une des nouvelles tendances de la théorie et de la pratique pédagogiques qui se basent sur l'analyse des processus de développement tant de l'enfance, de l'adolescence et de l'âge adulte. Car, selon Kotasek (1975), les nouvelles tendances de la théorie et de la pratique pédagogiques se caractérisent par leur refus d'une pédagogie centrée sur le sujet à enseigner (subject-centered) ou sur l'enfant (child-centered) et découlent de l'analyse des processus concrets de l'éducation chez l'élève, processus à dominante psychologique et sociologique, des divers stades d'évolution de l'enfance, de l'adolescence et de l'âge adulte (learningcentered education theory).

\section{Bibliographie}

Becker, H.S. Personal Change in Adult, Life-Sociometry, XXVII (1964), pp. 40-53.

Benaim, S. and Allen, I. (eds.) The Middle Years. London, England: T.V. Publications Limited, 1967.

Birren, J.E. The Psychology of Aging. Englewood Cliffs, N.J.: Prentice-Hall, 1964a.

Birren, J.E. (ed.) Relations of Development and Aging. Springfield, III: Charles C. Thomas, $1964 \mathrm{~b}$.

Bischof, L.J. Adult Psychology. N.Y.: Harper and Row, 1969.

Borow, H. (ed.) Man is a World at Work. Boston: Houghton Miffin, 1964.

Brim, O.S., Wheeler, S. Socialization after Childhood: Two Essays. N.Y.: John Wiley and Sons, 1966.

Bromley, D.B. The Psychology of Human Aging. Baltimore: Penguin, 1966. 
15 Vers un modèle du développement vocationnel de l'adulte

Brunne, E. et autres. An Overview of Adult Education Research. Chicago, III.: Adult Education Association of the U.S.A., 1969.

Bühler,.C. La psychologie dans la vie de notre temps. Paris: Privat Hachette, 1968.

Campbell, D, and Stanley, J.C. Experimental and Quasi-Experimental Designs for Research. Chicago: Rand McNally, 1963.

Castonguay, D. et autres. Rapport sur la formation continue. Québec, Association des Anciens de l'Université Laval, 1976.

Charters, A.N. Continuing Education for the Professions: in Handbook of Adult Education; Smith, R.M., Aker, G.F., et Kidd, J.R. (Eds.), The MacMillan Co., 1970, 487-499.

Chickering, Arthur W. Education E Identify. San Francisco: Jossey-Bass, 1969.

Clifford, G. and Odin, K. Young Adulthood: a Development Phase. College Studies in Social Work, vol. 44, no. $2,1974$.

Conseil Supérieur de l'Education. Le Collège: Rapport sur l'Etat et les Besoins de L'enseignement Collégial. Québec: M.E.Q., 1975.

Crites, J.O. Vocational Psychology. New York: McGraw-Hill, 1969.

Desmond, T.C. “America's Ynknown Middle-agers”, The New-York Times. July 29, 1956.

Dubois, J. L'analyse du discours, dans Marcellesi, J.B. (Ed.): Congrès de Tours: Etudes Socio-linguistiques. Paris: Le Pavillon, 1971.

Dubois, J. Scumpf, J. Un modèle d'enseignement du Français: Analyse Linguistique des Rapports d'Agrégation et du C.A.P.E.S., Langue française, no. 5, février 1970.

Dunaway, R. Youth into Maturity. N.Y.: Mental Health, Cox, Materials Center, 1970.

Erikson, Erik H. Youth, Change \& Challenge. N.Y.: Garden City, Doubleday, 1965.

Erikson, Erik H. Identity, Youth and Crisis. New-York: W.W. Norton \& Co. Inc., 1968.

Ferland, M. Cyril O. Houle et la Formation Continue Des Professionnels. Extension de l'Enseignement Universitaire. Université Laval: 1975.

Friedman, E. Changing Value Orientations in Adult Life. In Sociological Backgrounds of Adult Education, Burn, R.W. (ed.). N.Y.: Syracuse, CSLEA, 1970.

Ginzberg, et autres, Occupational Choice. New York: Columbia University Press, 1956.

Glass, J.C. et Rosenthal, P.H. Personality Changes in Adulthood: a Review of The Literature Australian, Journal of Adult Education, Vol. XIV, no. 2, juillet 1974, 65-69.

Goulet, L.R. and Baltes, P.B. Life-span Developmental Psychology Research and Theory. N.Y.: Academic Press, 1970.

Gould, R. Growth toward Self-Tolerance. Psychology Today, Feb. 1975, 74-78.

Hahn, M.E. Planning Ahead After Forty: The Process of Psycho-evaluation With Self-Study Projects. Calif.: Beverly Hills, Wester Psychological Services, 1967.

Havighurst, Robert J. Human Development and Education. New-York: McKay, 1969.

Havighurst, Robert J. Developmental Tasks and Education (3e ed.), New-York: David McKay Co., 1972.

Hendrickson, A. Adult Education, In: Encyclopedia of Education Research ( $3 \mathrm{e}$ ed.), C.W. Harris (Ed.). N.Y.: The MacMillan Company, 1960, pp. 3042.

Houle, Cyril O. The role of Continuing Education in Current Professional Development. American Library Association Bulletin, LXI (mars 1967), pp. 259-267.

Houle, Cyril O. Adult Education in Encyclopedia of Educational Research. 4th ed. New-York: McMillan, 1969, pp. 51-57.

Houle, Cyril O. The Educators of Adults, in Smith, R.M., Aker, G. F., Kidd, J.R. (Eds.), Handbook of Adult Education. N.Y.: MacMillan, 1970, 109-120.

Houle, Cyril O. The Changing Goals of Education in the Perspective of Lifelong Learning. International Review of Education (the Hogue), vol. 20, no. 4,1974, pp. 430-446.

Houle, Cyril O. Cyril O. Houle et la Formation Continue des Professionnels. Notes d'entrevues et 
morceaux choisis par Mario Ferland, Université Laval, Extension de l'enseignement Universitaire, 1975 .

Huberman, A.M. Permanent Education, Some Models of Adult Learning and adult change. Studies on permanent education, No. 22/1974, DECS 3/decs 6, Strasbourg, Janvier 1974.

Hurlock, E.B. Developmental Psychology (4e ed.), N.Y.: Mc Graw-Hill Book Company, 1975.

Hurtubise, René. L'Université Québécoise du Proche Avenir. Montréal: Hurtubise HMH, 1973.

Jordaan, Jean-Pierre. Life Stages as Organizing Modes of Career Development; in Vocational Guidance and Human Development, E.L. Herr (Ed.). Boston: Houghton, 1974, chp. II, 263-295.

Kaluger, G. and Kaluger, M.F. Profiles in Human Development. Saint-Louis: the C.O. Mosby Company, 1976.

Kotakek, J. La Formation de l'Enseignant, Problème Central de l'Education. Dans l'Education en Devenir. Paris: Les Presses de L'UNESCO, 1975.

Kreitlow, B.W. Research and theory. In Smith, R.M., Aker, G.F. Kidd, J.R. (Eds.), Handbook of Adult Education, N.Y.: MacMillan Co. 1970.

Kroll, A.M. Dinklage, L.B., Lee, J., Morley, E.D., Wilson, E.H. Career Development: Growth and Crisis. N.Y.: John Wiley and Sons, 1970.

Langis et ali. Liste des Projets de Développement Pédagogique des Organismes de l'Education des Adultes en 1973-74. Québec: Ministère de l'Education, 1974.

Lerner, J.O. Concepts and Theories of Human Development. London: Addison Wesley Publ. Comp., 1976.

Levinson, D.J., Darrow, C.M., Klein, E.B., Levinson, M.H. McKee, B. Periods in the Adult Development of Men: Ages 18 to 45. The Counseling Psychologist, Vol. 6, no. 1, 1976, pp. 21-25.

Linderman, E.C. The Meaning of Adult Education. Montréal: Harvest House, 1961.

Looft, W.R. Socialization and Personality Throughout the Life-span: an Examination of Contemporary Psychological Approaches. In Baltes P.B. et Schaie K.W. (Eds.), Life-Span Developmental Psychology: Personality and Socialization. N.Y.: Academic Press, 1973, pp. 26-53.

Nadeau, J.G. Le Collège: Rapport sur l'Etat et les Besoins de l'Enseignement Collégial. Québec: Bibliothèque Nationale, 3e trimestre, 1975.

Nesselroade, J.R., Reese, L.W. Life-span Developmental Psychology: Methodelogical Issues. N.Y.: Academic Press, 1973.

Neugarten, B.L. Middle Age and Aging. Chicago: The University of Chicago Press, 1975.

Neugarten, B.L. et autres. Age Norms, Age Constraints and Adult Socialization. American Journal of Sociology, LXX (1965), pp. 710-717.

O'Hara, R.P., Tiedeman, D.V. Vocational Self-concept in Adolescence, Journal of Counseling Psychology. Vol. 6, no. 4, 1959.

Overton, W.F., Reese, H.W. Models of Development: Methodelogical Implications. In Nesselroade, J.R. et Reese, H.W. (eds.); Life-Span Developmental Psychology: Methodological Issues. N.Y.: Academic Press, 1973.

Osipow, S.H. Theories of Career Development (2nd edition), New-York: Appleton-Century-Crofts, 1973.

Parker, C.A. Student Development: What Does It Mean? Journal of College Student Personnel, vol. 15 (4), juillet 1974.

Pécheux, M. Analyse Automatique du Discours. Coll. Sciences du comportement. DUNOD: Paris, 1969.

Pikunas, J. Human Development: a Science of Growth. N.Y.: McGraw-Hill Book Company, 1969.

Plante, P. Déredec. Logiciel pour le Traitement Linguistique et l'Analyse de Contenu des Textes. Document non publié, Montréal: Service de l'informatique de l'Université du Québec, Août 1977.

Prince, J. Identification and Analysis of Selected Developmental Task of College Students. Dissertation Abstracts, 1973-74: 4863. 
17 Vers un modèle du développement vocationnel de l'adulte

Rappoport, L. Adult Development: Faster Horses . . . and More money. Personal and Guidance Journal. 1976, 55(3), 106-112.

Riverin-Simard, D., Roberge-Brassard, J. Synthèse des Travaux de Recherche sur la Télé-Université Effectués par l'INRS-Education (1974-75) et Conjointement par ces Deux Organismes (1975-78). L'Orientation Professionnelle, Vol. 13, no. 4, Hiver 1977.

Robin, R. Histoire et Linguistique. Paris: Librairie Armand Collin, 1973.

Schwartz, B. L'éducation Demain. Paris: Aubin, Montaigne, 1973, Collection R.E.S.: (Recherches Economiques et Sociales).

Schaie, K.W. Methodological Problems in Descriptive Developmental Research on Adulthood and Aging. In Nesselroade J.R. et Reese T.W. (Eds.). Life-Span Developmental Psychology, Methodological Issues, N.Y.: Academic Press, 1973.

Schaie, K.W. and Gribbin, K. Adult Development and Aging. Annual Review of Psychology; Rosenzweig M.R. et Porter, L. M. (Eds.), vol. 26, 1975, pp. 65-97.

Schaie, K.W. Toward a Stage Theory of Adult Cognitive Development. J. Aging and Human Development, Vol. 8 (2), 1977-78.

Sheehy, G. Passages: Crises privisibles de l'âge adulte. Montréal: Editions Select, 1977.

Smith, Robert M., Aker, George F. and Kidd, J.R. Handbook of Adult Education. New-York: MacMillan, 1970.

Super, D.E. The Psychology of Careers. N.Y.: Harper and Row, 1957.

Super, D.E. Career Education and the Meaning of Work. Paper prepared for the Office of Career Education. Office of Education U.S. Department of Health, Education and Welfare, Final Draft, August 28, 1975. (sous presse).

Super, D.E. Stariskevsky, R., Matlin, N., Jordaan, J.P. Career Development: Self-Concept Theory. N.Y.: College Entrance Examination Board, 1963.

Time, July 1, 1966, pp. 60.

Tuffler, Alvin. Le choc du futur. Paris: Gonthier, 1970.

Tryon, C., Lilienthal, J.W. Developmental Tasks: The Concept and Importance in Fostering Mental Health in our Schools. Washington P.C.: National Education Association, 1950.

Vaillant, G.E. The Climb to Maturity: How the Best and the Brightest Came of Age. Psychology Today, sept. 1977.

Vedder, C.B. Problems of the Middle-Aged. Springfield, III.: Charles C. Thomas, 1965.

Vincent, E.L., Martin, P.C. Human Psychological Development. N.Y.: Ronald, 1961.

Wohlwill, J.F. Methodology and Research Strategy in the Study of Developmental Change. In Goulet L.R. et Baltes P.B. (Eds.): Life-Span Developmental Psychology. N.Y.: Academic Press, 1970, pp. 149-191. 